\title{
Characterizations of weighted dynamic Hardy-type inequalities with higher-order derivatives
}

\author{
S.H. Saker ${ }^{1,2}$, R.R. Mahmoud ${ }^{3,4^{*}}$ and K.R. Abdo ${ }^{3,4}$
}

\section{"Correspondence:}

ramy.ramadan.rus@cas.edu.om: rrm00@fayoum.edu.eg

${ }^{3}$ University of Technology and Applied Sciences-ALRustaq, 329Rustaq, Sultanate of Oman

${ }^{4}$ Department of Mathematics, Faculty of Science, Fayoum University, Fayoum, Egypt Full list of author information is available at the end of the article

\begin{abstract}
In this paper, we establish some necessary and sufficient conditions for the validity of a generalized dynamic Hardy-type inequality with higher-order derivatives with two different weighted functions on time scales. The corresponding continuous and discrete cases are captured when $\mathbb{T}=\mathbb{R}$ and $\mathbb{T}=\mathbb{N}$, respectively. Finally, some applications to our main result are added to conclude some continuous results known in the literature and some other discrete results which are essentially new.
\end{abstract}

MSC: 26A15;26D10; 39A13; 34N05

Keywords: Hardy's inequality; Higher-order derivatives; Time scales

\section{Introduction}

In [15] Hardy proved the classical continuous inequality

$$
\int_{0}^{\infty} \frac{1}{x}\left(\int_{0}^{x} f(t) d t\right)^{p} d x \leq\left(\frac{p}{p-1}\right)^{p} \int_{0}^{\infty} f^{p}(x) d x
$$

by using the calculus of variations in the twenties, where $f(x)$ is a positive integrable function over any finite interval $(0, x), f^{p}$ is an integrable convergent function over $(0, \infty)$, and $p>1$. Due to the importance of this inequality in mathematical and harmonic analysis, the extensions and generalizations have been studied by several authors, and various results have been established. We refer the reader to the papers $[2,3,5,10,17]$ and books $[16,24,25,28]$ that deal with these inequalities by discovering new proofs, generalizations, and extensions. For example, Muckenhoupt in [26] proved that the inequality

$$
\left[\int_{0}^{\infty}\left|u(x) \int_{0}^{x} f(t) d t\right|^{p} d x\right]^{1 / p} \leq C \quad\left[\int_{0}^{\infty}|f(x) v(x)|^{p} d x\right]^{1 / p}
$$

holds if and only if the following conditions hold:

$$
K=\sup _{r>0}\left(\int_{r}^{\infty}|u(x)|^{p} d x\right)^{1 / p}\left(\int_{0}^{r}|v(x)|^{-p^{\prime}} d x\right)^{1 / p^{\prime}}<\infty,
$$

(c) The Author(s) 2021. This article is licensed under a Creative Commons Attribution 4.0 International License, which permits use, sharing, adaptation, distribution and reproduction in any medium or format, as long as you give appropriate credit to the original author(s) and the source, provide a link to the Creative Commons licence, and indicate if changes were made. The images or other third party material in this article are included in the article's Creative Commons licence, unless indicated otherwise in a credit line to the material. If material is not included in the article's Creative Commons licence and your intended use is not permitted by statutory regulation or exceeds the permitted use, you will need to obtain permission directly from the copyright holder. To view a copy of this licence, visit http://creativecommons.org/licenses/by/4.0/. 
and $K \leq C \leq K(p)^{1 / p}\left(p^{\prime}\right)^{1 / p^{\prime}}$, where $1 / p+1 / p^{\prime}=1$ for $1 \leq p \leq \infty$. Opic et al. in [28] proved that the inequality holds

$$
\left(\int_{a}^{b} u(x)\left(\int_{a}^{x} f(t) d t\right)^{q} d x\right)^{1 / q} \leq C\left(\int_{a}^{b} f^{p}(x) v(x) d x\right)^{1 / p},
$$

where $-\infty \leq a<b \leq \infty, u, v$ are measurable positive functions in $(a, b)$, and $1<p \leq q<\infty$ if and only if

$$
A_{M}:=\sup _{a<r<b}\left(\int_{r}^{b} u(x) d x\right)^{1 / q}\left(\int_{a}^{b} v^{1-p^{\prime}}(x) d x\right)^{1 / p^{\prime}}<\infty .
$$

Moreover, the estimate for the constant $C$ in (1.1) is given by

$$
C \leq\left(1+\frac{q}{p^{\prime}}\right)^{1 / q}\left(1+\frac{p^{\prime}}{q}\right)^{1 / p^{\prime}} A_{M}
$$

In 1984 Gurka [11] proved that the following inequality, which contains the first-order derivative of $u$,

$$
\left(\int_{a}^{b}|u(x)|^{q} w(x) d x\right)^{1 / q} \leq C_{L}\left(\int_{a}^{b}\left|u^{\prime}(x)\right|^{p} v(x) d x\right)^{1 / p}
$$

holds for every $u \in A C(a, b)$ such that $u(a)=0$ if and only if

$$
B_{L}:=\sup _{a<x<b}\left(\int_{x}^{b} w(x) d x\right)^{1 / q}\left(\int_{a}^{x} v^{1-p^{\prime}}(t) d t\right)^{1 / p^{\prime}}<\infty
$$

and for the best possible constant $C_{L}$ in (1.2), the following estimate was satisfied: $B_{L} \leq$ $C_{L} \leq p^{1 / q}\left(p^{\prime}\right)^{1 / q^{\prime}} B_{L}$.

The classical Wirtinger inequality, see Hardy et al. [16, Theorem 257], is given by

$$
\int_{a}^{b}\left(u^{\prime}(t)\right)^{2} d t \geq \int_{a}^{b} u^{2}(t) d t
$$

for any $u \in C^{1}([a, b])$ satisfying $u(a)=u(b)=0$. Wirtinger-type inequalities are studied in the literature in both the continuous and discrete settings. In principle, it is an integral or sum estimate between the function and its derivative or difference, respectively. These types of inequalities have received a lot of attention because of their applications, for example, in number theory, especially in studies concerning the distribution of the zeros of the Riemann-zeta function [12-14]. In $[4,9,11]$ the authors studied some inequalities containing the first-order derivative with two different weighted functions. In [19], Hinton and Lewis extended inequality (1.3) and proved by using the Schwarz inequality that

$$
\int_{a}^{b} \frac{M^{2}(t)}{\left|M^{\prime}(t)\right|}\left(u^{\prime}(t)\right)^{2} d t \geq \frac{1}{4} \int_{a}^{b}\left|M^{\prime}(t)\right| u^{2}(t) d t
$$

for any positive function $M \in C^{1}([a, b])$ with $M^{\prime}(t) \neq 0, u \in C^{2}([a, b])$, and $u(a)=u(b)=0$. In [29], Peňa established the discrete analogue of (1.4) and proved the following result: For 
a positive sequence $\left\{M_{n}\right\}_{0 \leq n \leq N+1}$ satisfying either $\Delta M>0$ or $\Delta M<0$ on $[0, N] \cap \mathbb{Z}$,

$$
\sum_{n=0}^{N} \frac{M_{n} M_{n+1}}{\left|\Delta M_{n}\right|}\left(\Delta u_{n}\right)^{2} \geq \frac{1}{\psi_{J}} \sum_{n=0}^{N}\left|\Delta M_{n}\right| u_{n+1}^{2}
$$

holds for any sequence $\left\{u_{n}\right\}_{0 \leq n \leq N+1}$ with $u_{0}=u_{N+1}=0$, where

$$
\psi_{J}=\left(\sup _{0 \leq n \leq N} \frac{M_{n}}{M_{n+1}}\right)\left[1+\left(\sup _{0 \leq n \leq N} \frac{\left|\Delta M_{n}\right|}{\left|\Delta M_{n+1}\right|}\right)^{1 / 2}\right]^{2} .
$$

Stepanov in [44] was interested in inequalities containing higher-order derivative. In particular, he proved that for $1<p \leq q<\infty$ and $k \geq 1$ the inequality

$$
\left[\int_{0}^{\infty}|u(x)|^{q} \omega(x) d x\right]^{1 / q} \leq C\left[\int_{0}^{\infty}\left|u^{(k)}(x)\right|^{p} v(x) d x\right]^{1 / p}
$$

holds for all functions $u$ with $u^{(k-1)}$ locally absolutely continuous on $[0, \infty)$ and satisfies the condition

$$
u(0)=u^{\prime}(0)=\cdots=u^{(k-1)}(0)=0
$$

if and only if the following two conditions are fulfilled:

$$
\sup _{0<x<\infty}\left(\int_{x}^{\infty}(t-x)^{(k-1) q} \omega(t) d t\right)^{1 / q}\left(\int_{0}^{x} v^{1-p^{\prime}}(t) d t\right)^{1 / p^{\prime}}<\infty
$$

and

$$
\sup _{0<x<\infty}\left(\int_{x}^{\infty} \omega(t) d t\right)^{1 / q}\left(\int_{0}^{x}(x-t)^{(k-1) p^{\prime}} v^{1-p^{\prime}}(t) d t\right)^{1 / p^{\prime}}<\infty
$$

Kufner et al. [23] studied inequality (1.7) when $k=m+n$, and considered the inequality

$$
\left[\int_{0}^{\infty}|u(x)|^{q} \omega(x) d x\right]^{1 / q} \leq C\left[\int_{0}^{\infty}\left|u^{(m+n)}(x)\right|^{p} v(x) d x\right]^{1 / p}
$$

for any finite constant $C$ under the following conditions on $u$ :

$$
\begin{aligned}
& u(0)=u^{\prime}(0)=\cdots=u^{(m-1)}(0)=0, \\
& u^{(m)}(\infty)=u^{(m+1)}(\infty)=\cdots=u^{(m+n-1)}(\infty)=0, \quad m, n \geq 1 .
\end{aligned}
$$

To be more precise, they derived the necessary and sufficient conditions for the validity of this inequality (1.8) and proved that (1.8) holds if and only if

$$
B_{1}=\sup _{0<x<\infty}\left(\int_{x}^{\infty} \omega(t) t^{(m-1) q} d t\right)^{1 / q}\left(\int_{0}^{x}(v(t))^{1-p^{\prime}} t^{n p^{\prime}} d t\right)^{1 / p^{\prime}}<\infty
$$


and

$$
B_{2}=\sup _{0<x<\infty}\left(\int_{0}^{x} \omega(t) t^{m q} d t\right)^{1 / q}\left(\int_{x}^{\infty}(v(t))^{1-p^{\prime}} t^{(n-1) p^{\prime}} d t\right)^{1 / p^{\prime}}<\infty .
$$

For more results on the study of inequalities of higher-order derivative, we refer the reader to the papers $[21-23,43,44]$ and the references they cite.

In recent years, the dynamic inequalities on time scales, when the domain of the unknown function is a time scale $\mathbb{T}$, have been studied by several authors; we refer the reader to $[1,27,30,31,34-42]$ and the references they cite. Some of these papers dealt with the inequalities which have two weighted functions $u(x)$ and $v(x)$ and others dealt also with special examples of $u$ and $v$ as in $[32,33]$. Now we will recall some of these results that motivated the main aim of this paper. In [33] Saker et al. established the time scale version of dynamic inequality (1.1). They proved that the inequality

$$
\left(\int_{a}^{b} u(x)\left(\int_{a}^{\sigma(x)} f(t) \Delta t\right)^{q} \Delta x\right)^{1 / q} \leq C\left(\int_{a}^{b} f^{p}(x) v(x) \Delta x\right)^{1 / p}
$$

holds if and only if

$$
B:=\sup _{a<x<b}\left(\int_{x}^{b} u(t) \Delta t\right)^{1 / q}\left(\int_{a}^{\sigma(x)} v^{1-p^{\prime}}(t) \Delta t\right)^{1 / p^{\prime}}<\infty .
$$

Moreover, for the constant $C$ in (1.9), the following estimation is satisfied $B \leq C \leq k(p, q) B$, where $k(p, q)$ is defined by

$$
k(p, q)=\left(1+\frac{q}{p^{\prime}}\right)^{1 / q}\left(1+\frac{p^{\prime}}{q}\right)^{1 / p^{\prime}} \text { and } 1<p \leq q<\infty .
$$

In [18], Hilscher proved a Wirtinger-type inequality on time scales, which gives a unification of (1.4) and (1.5). In particular, he proved that if $M$ is a positive function and satisfies either $M^{\Delta}>0$ or $M^{\Delta}<0$, then

$$
\int_{a}^{b} \frac{M(t) M(\sigma(t))}{\left|M^{\Delta}(t)\right|}\left(y^{\Delta}(t)\right)^{2} \Delta t \geq \frac{1}{\psi^{2}} \int_{a}^{b}\left|M^{\Delta}(t)\right|\left(y^{\sigma}(t)\right)^{2} \Delta t
$$

holds for a positive function $y$ with $y(a)=y(b)=0$, and

$$
\psi=\left(\sup _{t \in \mathcal{I}^{\kappa}} \frac{M(t)}{M(\sigma(t))}\right)^{1 / 2}+\left[\left(\sup _{t \in \mathcal{I}^{\kappa}} \frac{\mu(t)\left|M^{\Delta}(t)\right|}{M(\sigma(t))}\right)+\left(\sup _{t \in \mathcal{I}^{\kappa}} \frac{M(t)}{M(\sigma(t))}\right)\right]^{1 / 2} .
$$

Following these trends and to develop the study of dynamic inequalities of Hardy-type of the differential forms on time scales, we prove the time scales version of the higher-order derivative inequality (1.8) on an arbitrary time scale $\mathbb{T}$.

The rest of the paper is organized as follows: In Sect. 2, we present some preliminaries about the theory of time scales and the time scales version of Fubini's theorem which is the cornerstone of our main proof. Also, we prove some essential prerequisite lemmas. In Sect. 3, we prove the main result of this paper (Theorem 3.1) which is a generalization of the weighted Hardy-type inequality with two different weights for a function which 
possesses higher-order derivatives. Next, we give some applications to our main results to capture some known results and to derive some new ones.

\section{Preliminaries and basic lemmas}

We suppose that the reader is familiar with time scales as presented in the monographs $[7,8]$. For the present paper to be self-contained, we only give here basic facts that are essentially used in the proofs of our results. For any function $f: \mathbb{T} \rightarrow \mathbb{R}$, where $\mathbb{T}$ is a time scale, the notation $f^{\sigma}(t)=f \circ \sigma(t)$ denotes the forward shift, where $\sigma$ stands for the forward jump operator and $f^{\Delta}$ denotes the delta derivative. For two $\Delta$-differentiable functions $f$ and $g$, their $\Delta$-derivative for the product is given by

$$
(f g)^{\Delta}(t)=f^{\Delta}(t) g(t)+f(\sigma(t)) g^{\Delta}(t) .
$$

The chain rule formula on time scales (Keller's chain rule) [7] is given by

$$
\left(x^{\gamma}(t)\right)^{\Delta}=\gamma \int_{0}^{1}\left[h x^{\sigma}+(1-h) x\right]^{\gamma-1} d h x^{\Delta}(t) .
$$

Throughout the paper, we assume that the functions in the statements of the theorems are nonnegative, $r d$-continuous functions and the integrals are assumed to exist. We next state Fubini's theorem due to Bibi et al. [6].

Theorem 2.1 Let $X$ and $Y$ be two time scales. If $: X \times Y \rightarrow \mathbb{R}$ is a $\Delta$-integrable function and if we define the functions

$$
\varphi(y)=\int_{X} f(x, y) \Delta x \quad \text { for a.e. } y \in Y
$$

and

$$
\psi(x)=\int_{Y} f(x, y) \Delta y \quad \text { for a.e. } x \in X,
$$

then $\varphi$ is $\Delta$-integrable on $Y$ and $\psi$ is $\Delta$-integrable on $X$ and

$$
\int_{X} \Delta x \int_{Y} f(x, y) \Delta y=\int_{Y} \Delta y \int_{X} f(x, y) \Delta x .
$$

In the following, we prove the basic inequalities that will be used to prove the main results by using Keller's chain rule and some concepts on time scales.

Lemma 2.1 If $m, n \geq 1$ and

$$
k_{1}(x, s)=\int_{0}^{s}(x-t)^{m-1}(s-t)^{n-1} \Delta t,
$$

then

$$
\frac{1}{(m+n-1)} \leq x^{-m+1} s^{-n} k_{1}(x, s) \leq \frac{1}{n},
$$

where $0<t \leq \sigma(t)<s<x$. 
Proof From the definition of $k_{1}(x, s)$ and since $0<t \leq \sigma(t)<s<x$, we see that

$$
\begin{aligned}
k_{1}(x, s) & =\int_{0}^{s}(x-t)^{m-1}(s-t)^{n-1} \Delta t \leq \int_{0}^{s} x^{m-1}(s-t)^{n-1} \Delta t \\
& =x^{m-1} \int_{0}^{s}(s-t)^{n-1} \Delta t
\end{aligned}
$$

Applying Keller's chain rule (2.1) on the right-hand side of equation (2.5), we obtain that

$$
\left((s-t)^{n}\right)^{\Delta}=n \int_{0}^{1}((1-h)(s-t)+h(s-\sigma(t)))^{n-1} d h(s-t)^{\Delta} .
$$

Using the definition of forward jump operator, we see that

$$
\left((s-t)^{n}\right)^{\Delta} \leq-n \int_{0}^{1}((1-h+h)(s-t))^{n-1} d h=-n[s-t]^{n-1} .
$$

Dividing both sides by $(-n)$, we find that

$$
(s-t)^{n-1} \leq-\frac{1}{n}\left((s-t)^{n}\right)^{\Delta}
$$

Substituting (2.6) into (2.5), we obtain that

$$
\begin{aligned}
k_{1}(x, s) & \leq x^{m-1}\left(\frac{-1}{n} \int_{0}^{s}\left((s-t)^{n}\right)^{\Delta} \Delta t\right)=-\frac{1}{n} x^{m-1}\left[(s-t)^{n}\right]_{t=0}^{t=s} \\
& =-\frac{1}{n} x^{m-1}\left[(s-s)^{n}-(s-0)^{n}\right]=\frac{1}{n} x^{m-1} s^{n}
\end{aligned}
$$

which leads directly to

$$
x^{-m+1} s^{-n} k_{1}(x, s) \leq \frac{1}{n} \text {. }
$$

Since $t \leq \sigma(t)<s<x$, we can write that

$$
\frac{t}{s}>\frac{t}{x} \quad \Longrightarrow \quad 1-\frac{t}{s}<1-\frac{t}{x} .
$$

We will deal now with the lower bound of $k_{1}(x, s)$. Multiplying both sides of (2.3) by the term $x^{-m+1} s^{-n}$, we obtain that

$$
\begin{aligned}
x^{-m+1} s^{-n} k_{1}(x, s) & =\int_{0}^{s}(x-t)^{m-1}(s-t)^{n-1} x^{-m+1} s^{-n} \Delta t \\
& =\frac{1}{s} \int_{0}^{s}\left(\frac{x-t}{x}\right)^{m-1}\left(\frac{s-t}{s}\right)^{n-1} \Delta t \\
& =\frac{1}{s} \int_{0}^{s}\left(1-\frac{t}{x}\right)^{m-1}\left(1-\frac{t}{s}\right)^{n-1} \Delta t .
\end{aligned}
$$

Using condition (2.8) into equation (2.9), we have that

$$
x^{-m+1} s^{-n} k_{1}(x, s) \geq \frac{1}{s} \int_{0}^{s}\left(1-\frac{t}{s}\right)^{m-1}\left(1-\frac{t}{s}\right)^{n-1} \Delta t
$$




$$
=\frac{1}{s} \int_{0}^{s}\left(1-\frac{t}{s}\right)^{m+n-2} \Delta t
$$

Applying Keller's chain rule (2.1) on the right-hand side of equation (2.10), we get that

$$
\begin{aligned}
& \left(\left(1-\frac{t}{s}\right)^{m+n-1}\right)^{\Delta} \\
& =(m+n-1) \int_{0}^{1}\left[(1-h)\left(1-\frac{t}{s}\right)+h\left(1-\frac{\sigma(t)}{s}\right)\right]^{m+n-2} d h\left(1-\frac{t}{s}\right)^{\Delta} \\
& \geq-\frac{1}{s}(m+n-1) \int_{0}^{1}\left((1-h+h)\left(1-\frac{t}{s}\right)\right)^{m+n-2} d h
\end{aligned}
$$

which gives that

$$
\begin{aligned}
& \left(\left(1-\frac{t}{s}\right)^{m+n-1}\right)^{\Delta} \geq-\frac{1}{s}(m+n-1)\left(1-\frac{t}{s}\right)^{m+n-2} \\
& -\frac{1}{m+n-1}\left[\left(1-\frac{t}{s}\right)^{m+n-1}\right]^{\Delta} \leq \frac{1}{s}\left(1-\frac{t}{s}\right)^{m+n-2}
\end{aligned}
$$

Integrating both sides of inequality (2.11) and taking into account the fact (2.10), we have that

$$
-\frac{1}{m+n-1}\left[\left(1-\frac{t}{s}\right)^{m+n-1}\right]_{t=0}^{t=s} \leq \frac{1}{s} \int_{0}^{s}\left(1-\frac{t}{s}\right)^{m+n-2} \Delta t
$$

and therefore

$$
x^{-m+1} s^{-n} k_{1}(x, s) \geq \frac{1}{m+n-1} .
$$

Consequently, from (2.7) and (2.12), we get the desired result (2.4). This completes the proof.

Lemma 2.2 If $m, n \geq 1$, and

$$
k_{2}(x, s)=\int_{0}^{x}(x-t)^{m-1}(s-t)^{n-1} \Delta t
$$

then

$$
\frac{1}{m+n-1} \leq x^{-m} s^{-n+1} k_{2}(x, s) \leq \frac{1}{m}
$$

where $0<t \leq \sigma(t)<x<s$.

Proof From the definition of $k_{2}(x, s)$ and since $0<t \leq \sigma(t)<x<s$, we have that

$$
\begin{aligned}
k_{2}(x, s) & =\int_{0}^{x}(x-t)^{m-1}(s-t)^{n-1} \Delta t \leq \int_{0}^{x}(x-t)^{m-1} s^{n-1} \Delta t \\
& =s^{n-1} \int_{0}^{x}(x-t)^{m-1} \Delta t .
\end{aligned}
$$


Applying Keller's chain rule (2.1) on the right-hand side of equation (2.15), we obtain that

$$
\left((x-t)^{m}\right)^{\Delta}=m \int_{0}^{1}((1-h)(x-t)+h(x-\sigma(t)))^{m-1} d h(-1) .
$$

This implies that

$$
\begin{aligned}
\left((x-t)^{m}\right)^{\Delta} & \leq-m \int_{0}^{1}((1-h+h)(x-t))^{m-1} d h \\
& =-m(x-t)^{m-1} \geq\left((x-t)^{m}\right)^{\Delta} .
\end{aligned}
$$

So that

$$
(x-t)^{m-1} \leq-\frac{1}{m}\left((x-t)^{m}\right)^{\Delta} .
$$

Substituting (2.16) into (2.15), we obtain

$$
\begin{aligned}
k_{2}(x, s) & \leq-\frac{s^{n-1}}{m} \int_{0}^{x}\left((x-t)^{m}\right)^{\Delta} \Delta t=-\frac{1}{m} s^{n-1}\left[(x-t)^{m}\right]_{t=0}^{t=x} \\
& =-\frac{1}{m} s^{n-1}\left(0-x^{m}\right)=\frac{1}{m} s^{n-1} x^{m},
\end{aligned}
$$

that is,

$$
x^{-m} s^{-n+1} k_{2}(x, s) \leq \frac{1}{m} .
$$

By using the fact that $t \leq \sigma(t)<x<s$, we get that

$$
1-\frac{t}{x}<1-\frac{t}{s}
$$

We will deal with equation (2.13), since

$$
k_{2}(x, s)=\int_{0}^{x}(x-t)^{m-1}(s-t)^{n-1} \Delta t
$$

then multiplying both sides of the last equation by the term $x^{-m} s^{-n+1}$, we obtain that

$$
\begin{aligned}
x^{-m} s^{-n+1} k_{2}(x, s) & =\int_{0}^{x}(x-t)^{m-1}(s-t)^{n-1} s^{-n+1} x^{-m} \Delta t \\
& =\frac{1}{x} \int_{0}^{x}\left(\frac{x-t}{x}\right)^{m-1}\left(\frac{s-t}{s}\right)^{n-1} \Delta t \\
& =\frac{1}{x} \int_{0}^{x}\left(1-\frac{t}{x}\right)^{m-1}\left(1-\frac{t}{s}\right)^{n-1} \Delta t .
\end{aligned}
$$

By substituting (2.18) into equation (2.19), we have that

$$
x^{-m} s^{-n+1} k_{2}(x, s) \geq \frac{1}{x} \int_{0}^{x}\left(1-\frac{t}{x}\right)^{m-1}\left(1-\frac{t}{x}\right)^{n-1} \Delta t,
$$


which gives that

$$
x^{-m} s^{-n+1} k_{2}(x, s) \geq \frac{1}{x} \int_{0}^{x}\left(1-\frac{t}{x}\right)^{m+n-2} \Delta t .
$$

Applying Keller's chain rule (2.1) on the right-hand side of equation (2.20), we obtain that

$$
\begin{aligned}
& \left(\left(1-\frac{t}{x}\right)^{m+n-1}\right)^{\Delta} \\
& =(m+n-1) \int_{0}^{1}\left((1-h)\left(1-\frac{t}{x}\right)+h\left(1-\frac{\sigma(t)}{x}\right)\right)^{m+n-2} d h\left(-\frac{1}{x}\right) \\
& \geq-\frac{1}{x}(m+n-1) \int_{0}^{1}\left((1-h+h)\left(1-\frac{t}{x}\right)\right)^{m+n-2} d h \\
& =-\frac{1}{x}(m+n-1)\left(1-\frac{t}{x}\right)^{m+n-2} .
\end{aligned}
$$

That is,

$$
\left(\left(1-\frac{t}{x}\right)^{m+n-1}\right)^{\Delta} \geq-\frac{1}{x}(m+n-1)\left(1-\frac{t}{x}\right)^{m+n-2},
$$

and then

$$
-\frac{1}{m+n-1}\left[\left(1-\frac{t}{x}\right)^{m+n-1}\right]^{\Delta} \leq \frac{1}{x}\left(1-\frac{t}{x}\right)^{m+n-2} .
$$

Integrating both sides of inequality (2.21), we have that

$$
-\frac{1}{m+n-1}\left[\left(1-\frac{t}{x}\right)^{m+n-1}\right]_{t=0}^{t=x} \leq \frac{1}{x} \int_{0}^{x}\left(1-\frac{t}{x}\right)^{m+n-2} \Delta t .
$$

Substituting into (2.20), we obtain that

$$
x^{-m} s^{-n+1} k_{2}(x, s) \geq \frac{1}{m+n-1} .
$$

Consequently, from (2.17) and (2.22), we obtain the required result (2.14). This completes the proof.

\section{Main results and applications}

Now, we are in a position to state and prove our main results which assert the validity of the dynamic Hardy-type inequality for functions with higher-order $\Delta$-derivatives embedded with two different weighted functions. For simplicity, we will use the notations

$$
\begin{aligned}
& B_{1}=\sup _{x \in[0, \infty)_{\mathbb{T}}}\left(\int_{x}^{\infty} \omega(t) t^{(m-1) q} \Delta t\right)^{1 / q}\left(\int_{0}^{\sigma(x)} v^{1-p^{\prime}} t^{n p^{\prime}} \Delta t\right)^{1 / p^{\prime}}, \\
& B_{2}=\sup _{x \in[0, \infty)_{\mathbb{T}}}\left(\int_{0}^{\sigma(x)} \omega(t) t^{m q} \Delta t\right)^{1 / q}\left(\int_{x}^{\infty} v^{1-p^{\prime}} t^{(n-1) p^{\prime}} \Delta t\right)^{1 / p^{\prime}},
\end{aligned}
$$


and the boundary conditions

$$
\begin{aligned}
& u(0)=u^{\Delta}(0)=\cdots=u^{\Delta^{(m-1)}}(0)=0, \\
& u^{\Delta^{(m)}}(\infty)=u^{\Delta^{(m+1)}}(\infty)=\cdots=u^{\Delta^{(k-1)}}(\infty)=0,
\end{aligned}
$$

where $k=m+n, m$ and $n$ are nonnegative integers.

Theorem 3.1 Let $\mathbb{T}$ be a time scale with $1<p \leq q<\infty, u \in C_{r d}\left([0, \infty)_{\mathbb{T}}, \mathbb{R}^{+}\right)$, and $\omega$, $v$ are positive rd-continuous functions defined on $[0, \infty)_{\mathbb{T}}$. Then there exists a positive constant C such that the inequality

$$
\left[\int_{0}^{\infty}|u(x)|^{q} \omega(x) \Delta x\right]^{1 / q} \leq C\left[\int_{0}^{\infty}\left|u^{\Delta^{(m+n)}}(x)\right|^{p} v(x) \Delta x\right]^{1 / p}
$$

holds for every $u \in C_{r d}^{(m+n)}\left([0, \infty)_{\mathbb{T}}, \mathbb{R}^{+}\right)$if and only if $B_{1}<\infty$ and $B_{2}<\infty$.

Proof We shall show that conditions (3.1) and (3.2) are necessary and sufficient for (3.4) to hold. For simplicity, inequality (3.4) can take the following form, where $u=T f$ and $f=u^{\Delta^{(m+n)}}$ :

$$
\left[\int_{0}^{\infty}|(T f)(x)|^{q} \omega(x) \Delta x\right]^{1 / q} \leq C\left[\int_{0}^{\infty}|f(x)|^{p} v(x) \Delta x\right]^{1 / p} .
$$

Now, we will fix $m, n \geq 1$ and take $(T f)(x)$ as the form

$$
(T f)(x)=\frac{1}{(m-1) !(n-1) !} \int_{0}^{\sigma(x)}(x-t)^{m-1}\left[\int_{t}^{\infty}(s-t)^{n-1} f(s) \Delta s\right] \Delta t .
$$

Set $C_{m, n}=(m-1) !(n-1) !$, then we have

$$
\begin{aligned}
C_{m, n}(T f)(x)= & \int_{0}^{\sigma(x)}(x-t)^{m-1}\left[\int_{t}^{\infty}(s-t)^{n-1} f(s) \Delta s\right] \Delta t . \\
= & \int_{0}^{\sigma(x)}(x-t)^{m-1}\left[\int_{t}^{x}(s-t)^{n-1} f(s) \Delta s\right. \\
& \left.+\int_{x}^{\infty}(s-t)^{n-1} f(s) \Delta s\right] \Delta t \\
= & \int_{0}^{\sigma(x)}(x-t)^{m-1}\left[\int_{t}^{x}(s-t)^{n-1} f(s) \Delta s\right] \Delta t \\
& +\int_{0}^{x}(x-t)^{m-1}\left[\int_{x}^{\infty}(s-t)^{n-1} f(s) \Delta s\right] \Delta t .
\end{aligned}
$$

Now, by using Fubini's theorem on time scales (2.2), we have

$$
\begin{aligned}
C_{m, n}(T f)(x)= & \int_{0}^{\sigma(x)} f(s)\left[\int_{0}^{s}(x-t)^{m-1}(s-t)^{n-1} \Delta t\right] \Delta s \\
& +\int_{x}^{\infty} f(s)\left[\int_{0}^{x}(x-t)^{m-1}(s-t)^{n-1} \Delta t\right] \Delta s,
\end{aligned}
$$


then

$$
C_{m, n}(T f)(x):=\left(J_{1} f\right)(x)+\left(J_{2} f\right)(x),
$$

where

$$
\left(J_{1} f\right)(x)=\int_{0}^{\sigma(x)} k_{1}(x, s) f(s) \Delta s
$$

and

$$
\left(J_{2} f\right)(x)=\int_{x}^{\infty} k_{2}(x, s) f(s) \Delta s
$$

From (2.4) the function in (3.8) is equivalent to the function

$$
\int_{0}^{\sigma(x)} x^{m-1} s^{n} f(s) \Delta s
$$

Therefore from Hardy's inequality (1.9), and replacing $s^{n} f(s)$ with $\widetilde{f}(s), x^{(m-1) q} \omega(x)$ with $\widetilde{\omega}(x), x^{-n p} v(x)$ with $\widetilde{v}(x)$, and $x^{n} f(x)$ with $\widetilde{f}(x)$, we obtain that

$$
\begin{aligned}
& \left(\int_{0}^{\infty}\left(\int_{0}^{\sigma(x)} \tilde{f}(s) \Delta s\right)^{q} \widetilde{\omega}(x) \Delta x\right)^{1 / q} \\
& \quad=\left(\int_{0}^{\infty} \omega(x)\left(\int_{0}^{\sigma(x)} x^{m-1} s^{n} f(s) \Delta s\right)^{q} \Delta x\right)^{1 / q} \\
& \quad=\left(\int_{0}^{\infty}\left(\int_{0}^{\sigma(x)} s^{n} f(s) \Delta s\right)^{q} x^{(m-1) q} \omega(x) \Delta x\right)^{1 / q} \\
& \leq C\left(\int_{0}^{\infty} f^{p}(x) v(x) \Delta x\right)^{1 / p}=C\left(\int_{0}^{\infty} f^{p}(x) x^{n p} x^{-n p} v(x) \Delta x\right)^{1 / p} \\
& \quad=C\left(\int_{0}^{\infty}\left(x^{n} f(x)\right)^{p} \widetilde{v}(x) \Delta x\right)^{1 / p}=C\left(\int_{0}^{\infty}(\widetilde{f}(x))^{p} \widetilde{v}(x) \Delta x\right)^{1 / p} .
\end{aligned}
$$

Then

$$
\left[\int_{0}^{\infty}\left(\int_{0}^{\sigma(x)} \widetilde{f}(s) \Delta s\right)^{q} \widetilde{\omega}(x) \Delta x\right]^{1 / q} \leq C\left(\int_{0}^{\infty}(\widetilde{f}(x))^{p} \widetilde{v}(x) \Delta x\right)^{1 / p} .
$$

According to the same inequality (1.9), inequality (3.10) holds if and only if

$$
\begin{aligned}
B_{1} & =\sup _{0<x<\infty}\left(\int_{x}^{\infty} \widetilde{\omega}(t) \Delta t\right)^{1 / q}\left(\int_{0}^{\sigma(x)}(\widetilde{v}(t))^{1-p^{\prime}}(t) \Delta t\right)^{1 / p^{\prime}} \\
& =\sup _{0<x<\infty}\left(\int_{x}^{\infty} \omega(t) t^{(m-1) q} \Delta t\right)^{1 / q}\left(\int_{0}^{\sigma(x)}\left(t^{-n p} v\right)^{1-p^{\prime}} \Delta t\right)^{1 / p^{\prime}} \\
& =\sup _{0<x<\infty}\left(\int_{x}^{\infty} \omega(t) t^{(m-1) q} \Delta t\right)^{1 / q}\left(\int_{0}^{\sigma(x)} v^{1-p^{\prime}}(t) t^{n p^{\prime}} \Delta t\right)^{1 / p^{\prime}}<\infty,
\end{aligned}
$$


where $p^{\prime}=p /(p-1)$. From (2.14) the function in (3.9) is equivalent to the function

$$
\int_{x}^{\infty} s^{n-1} x^{m} f(s) \Delta s
$$

Therefore from Hardy's inequality (1.9) and replacing $s^{n-1} f(s)$ with $\bar{f}(s), x^{m q} \omega(x)$ with $\bar{\omega}(x)$, $x^{-(n-1) p} v(x)$ with $\bar{v}(x)$, and $x^{n-1} f(x)$ with $\bar{f}(x)$, we obtain

$$
\begin{aligned}
& \left(\int_{0}^{\infty}\left(\int_{x}^{\infty} \bar{f}(s) \Delta s\right)^{q} \bar{\omega}(x) \Delta x\right)^{1 / q} \\
& \quad=\left(\int_{0}^{\infty}\left(\int_{x}^{\infty} s^{n-1} x^{m} f(s) \Delta s\right)^{q} \omega(x) \Delta x\right)^{1 / q} \\
& \quad=\left(\int_{0}^{\infty} \int_{x}^{\infty}\left(s^{n-1} f(s) \Delta s\right)^{q} x^{m q} \omega(x) \Delta x\right)^{1 / q} \\
& \quad \leq C\left(\int_{0}^{\infty} f^{p}(x) v(x) \Delta x\right)^{1 / p}=C\left(\int_{0}^{\infty} f^{p}(x) x^{(n-1) p} x^{(-n+1) p} v(x) \Delta x\right)^{1 / p} \\
& \quad=C\left(\int_{0}^{\infty}\left(x^{n-1} f(x)\right)^{p} x^{(-n+1) p} v(x) \Delta x\right)^{1 / p}=C\left(\int_{0}^{\infty}(\bar{f}(x))^{p} \bar{v}(x) \Delta x\right)^{1 / p} .
\end{aligned}
$$

Then

$$
\left(\int_{0}^{\infty}\left(\int_{x}^{\infty} \bar{f}(s) \Delta s\right)^{q} \bar{\omega}(x) \Delta x\right)^{1 / q} \leq C\left(\int_{0}^{\infty}(\bar{f}(x))^{p} \bar{v}(x) \Delta x\right)^{1 / p} .
$$

According to the dual of inequality (1.9), this inequality holds if and only if

$$
\begin{aligned}
B_{2} & =\sup _{0<x<\infty}\left(\int_{0}^{\sigma(x)} \bar{\omega}(t) \Delta t\right)^{1 / q}\left(\int_{x}^{\infty}(\bar{v}(t))^{1-p^{\prime}} \Delta t\right)^{1 / p^{\prime}} \\
& =\sup _{0<x<\infty}\left(\int_{0}^{\sigma(x)} t^{m q} \omega(t) \Delta t\right)^{1 / q}\left(\int_{x}^{\infty}\left(t^{-(n-1) p} v(t)\right)^{1-p^{\prime}} \Delta t\right)^{1 / p^{\prime}} \\
& =\sup _{0<x<\infty}\left(\int_{0}^{\sigma(x)} t^{m q} \omega(t) \Delta t\right)^{1 / q}\left(\int_{x}^{\infty} v^{1-p^{\prime}}(t) t^{(n-1) p^{\prime}} \Delta t\right)^{1 / p^{\prime}}<\infty .
\end{aligned}
$$

So, we have shown that conditions (3.1) and (3.2) are necessary and sufficient for the validity of inequalities (3.10) and (3.11). As a result of (3.7), it follows directly that these conditions are also necessary and sufficient for inequality (3.5) which is equivalent to the required one (3.4). This completes the proof.

Remark 3.1 In Theorem 3.1, if we take $\mathbb{T}=\mathbb{R}$, we get the following continuous weighted Hardy inequality as mentioned in [23] and [28]:

$$
\left[\int_{0}^{\infty}|u(x)|^{q} \omega(x) d x\right]^{1 / q} \leq C\left[\int_{0}^{\infty}\left|u^{(m+n)}(x)\right|^{p} v(x) d x\right]^{1 / p},
$$

which will be satisfied if and only if the following conditions are satisfied:

$$
B_{3}=\sup _{x \in[0, \infty)}\left(\int_{x}^{\infty} \omega(t) t^{(m-1) q} d t\right)^{1 / q}\left(\int_{0}^{x} v^{1-p^{\prime}} t^{n p^{\prime}} d t\right)^{1 / p^{\prime}}<\infty
$$


and

$$
B_{4}=\sup _{x \in[0, \infty)}\left(\int_{0}^{x} \omega(t) t^{m q} d t\right)^{1 / q}\left(\int_{x}^{\infty} v^{1-p^{\prime}} t^{(n-1) p^{\prime}} d t\right)^{1 / p^{\prime}}<\infty .
$$

Remark 3.2 In Theorem 3.1, if we take $\mathbb{T}=\mathbb{N}$, we get the discrete analogue of inequality

$$
\left(\sum_{n=1}^{\infty} \omega_{n}\left(\sum_{k=1}^{n} a_{k}\right)^{q}\right)^{\frac{1}{q}} \leq C\left(\sum_{n=1}^{\infty} v_{n}\left(\Delta^{(k)} a_{n}\right)^{p}\right)^{1 / p}
$$

which will be satisfied if and only if

$$
B_{5}=\sup _{n}\left(\sum_{n=k}^{\infty} \omega_{n} n^{(m-1) q}\right)^{1 / q}\left(\sum_{k=1}^{n} v_{k}^{1-p^{\prime}} k^{n p^{\prime}}\right)^{1 / p^{\prime}}<\infty
$$

and

$$
B_{6}=\sup _{n}\left(\sum_{k=1}^{n} \omega_{k} k^{m q}\right)^{1 / q}\left(\sum_{n=k}^{\infty} v_{n}^{1-p^{\prime}} n^{(n-1) p^{\prime}}\right)^{1 / p^{\prime}}<\infty
$$

where $\Delta^{(k)} a_{n}=\Delta\left(\Delta^{(k-1)} a_{n}\right)$ and $\Delta a_{n}=a_{n+1}-a_{n}$. To the best of the authors' knowledge, this Hardy-type inequality for higher differences is essentially new.

In the rest of this section, we present some applications by making suitable substitutions for the two weighted functions $\omega(x)$ and $v(x)$. In the sequel, the constant $C$ may take different values not necessary to be the same. We start with the following consequence of the dynamic Hardy-type inequality.

Corollary 3.1 Let $\mathbb{T}$ be a time scale with $1<p \leq q<\infty, u \in C_{r d}\left([0, \infty)_{\mathbb{T}}, \mathbb{R}^{+}\right)$. Then there exists a positive constant $C$ such that the inequality

$$
\left[\int_{0}^{\infty}|u(x)|^{q} x^{\alpha} \Delta x\right]^{1 / q} \leq C\left[\int_{0}^{\infty}\left|u^{\Delta^{(m+n)}}(x)\right|^{p} x^{\beta} \Delta x\right]^{1 / p}
$$

holds for every $u$ if and only if

$$
B_{7}=\sup _{x \in[0, \infty)_{\mathbb{T}}}\left(\int_{x}^{\infty} t^{\alpha+(m-1) q} \Delta t\right)^{1 / q}\left(\int_{0}^{\sigma(x)} t^{\beta\left(1-p^{\prime}\right)+n p^{\prime}} \Delta t\right)^{1 / p^{\prime}}<\infty
$$

and

$$
B_{8}=\sup _{x \in[0, \infty)_{\mathbb{T}}}\left(\int_{0}^{\sigma(x)} t^{\alpha+m q} \Delta t\right)^{1 / q}\left(\int_{x}^{\infty} t^{\beta\left(1-p^{\prime}\right)+(n-1) p^{\prime}} \Delta t\right)^{1 / p^{\prime}}<\infty
$$

for some positive constants $\alpha, \beta$ and $m, n \geq 1$. 
Proof If we set $\omega(x)=x^{\alpha}$ and $v(x)=x^{\beta}$ in Theorem 3.1, we get the required result. This completes the proof.

Remark 3.3 In inequality (3.14), if we take $\mathbb{T}=\mathbb{R}$, we get the following continuous weighted inequality:

$$
\left[\int_{0}^{\infty}|u(x)|^{q} x^{\alpha} d x\right]^{1 / q} \leq C\left[\int_{0}^{\infty}\left|u^{(m+n)}(x)\right|^{p} x^{\beta} d x\right]^{1 / p}
$$

due to Kufner [23], which will be satisfied if and only if

$$
B_{9}=\sup _{x \in[0, \infty)}\left(\int_{x}^{\infty} t^{\alpha+(m-1) q} d t\right)^{1 / q}\left(\int_{0}^{x} t^{\beta\left(1-p^{\prime}\right)+n p^{\prime}} d t\right)^{1 / p^{\prime}}<\infty
$$

and

$$
B_{10}=\sup _{x \in[0, \infty)}\left(\int_{0}^{x} t^{\alpha+m q} d t\right)^{1 / q}\left(\int_{x}^{\infty} t^{\beta\left(1-p^{\prime}\right)+(n-1) p^{\prime}} d t\right)^{1 / p^{\prime}}<\infty
$$

for some positive constants $\alpha, \beta$.

Remark 3.4 In inequality (3.14), if we take $\mathbb{T}=\mathbb{N}$, we get the discrete analogue of inequality (3.14)

$$
\left(\sum_{n=1}^{\infty} n^{\alpha}\left(\sum_{k=1}^{n} a_{k}\right)^{q}\right)^{1 / q} \leq C\left(\sum_{n=1}^{\infty} n^{\beta}\left(\Delta^{(k)} a_{n}\right)^{p}\right)^{1 / p}
$$

which will be satisfied if and only if

$$
B_{11}=\sup _{n}\left(\sum_{n=k}^{\infty} n^{\alpha+(m-1) q}\right)^{1 / q}\left(\sum_{k=1}^{n} k^{\beta\left(1-p^{\prime}\right)+n p^{\prime}}\right)^{1 / p^{\prime}}<\infty
$$

and

$$
B_{12}=\sup _{n}\left(\sum_{k=1}^{n} k^{\alpha+m q}\right)^{1 / q}\left(\sum_{n=k}^{\infty} n^{\beta\left(1-p^{\prime}\right)+(n-1) p^{\prime}}\right)^{1 / p^{\prime}}<\infty
$$

which is essentially new.

Corollary 3.2 Let $\mathbb{T}$ be a time scale with $1<p<\infty$ and $u \in C_{r d}\left([0, \infty)_{\mathbb{T}}, \mathbb{R}^{+}\right)$. Then there exists a positive constant $C$ such that the inequality

$$
\int_{0}^{\infty}|u(x)|^{p} x^{\alpha-2 p} \Delta x \leq C \int_{0}^{\infty}\left|u^{(2)}(x)\right|^{p} x^{\alpha} \Delta x
$$

holds for every function $u$ if and only if

$$
B_{13}=\sup _{x \in[0, \infty)_{\mathbb{T}}}\left(\int_{x}^{\infty} t^{\alpha-2 p} \Delta t\right)^{1 / p}\left(\int_{0}^{\sigma(x)} t^{\alpha+p^{\prime}(1-\alpha)} \Delta t\right)^{1 / p^{\prime}}<\infty
$$


and

$$
B_{14}=\sup _{x \in[0, \infty)_{\mathbb{T}}}\left(\int_{0}^{\sigma(x)} t^{\alpha-p} \Delta t\right)^{1 / p}\left(\int_{x}^{\infty} t^{\alpha\left(1-p^{\prime}\right)} \Delta t\right)^{1 / p^{\prime}}<\infty
$$

for some positive constant $\alpha$ and $u(0)=u^{\Delta}(\infty)=0$.

Proof If we take $\omega(x)=x^{\alpha-2 p}, v(x)=x^{\alpha}, m=n=1$ for the case $p=q$ in inequality (3.4), we will obtain the required result. This completes the proof.

Remark 3.5 In inequality (3.15), if we take $\mathbb{T}=\mathbb{R}$, we get the following continuous weighted inequality due to Kufner [25] for the inequality

$$
\int_{0}^{\infty}|u(x)|^{p} x^{\alpha-2 p} d x \leq C \int_{0}^{\infty}\left|u^{\prime \prime}(x)\right|^{p} x^{\alpha} d x
$$

which will be satisfied if and only if

$$
B_{15}=\sup _{x \in[0, \infty)}\left(\int_{x}^{\infty} t^{\alpha-2 p} d t\right)^{1 / p}\left(\int_{0}^{x} t^{\alpha+p^{\prime}(1-\alpha)} d t\right)^{1 / p^{\prime}}<\infty
$$

and

$$
B_{16}=\sup _{x \in[0, \infty)}\left(\int_{0}^{x} t^{\alpha-p} d t\right)^{1 / p}\left(\int_{x}^{\infty} t^{\alpha\left(1-p^{\prime}\right)} d t\right)^{1 / p^{\prime}}<\infty
$$

for some positive constant $\alpha$ and $u(0)=u^{\prime}(\infty)=0$.

Remark 3.6 In inequality (3.14), if we take $\mathbb{T}=\mathbb{N}$, we get the discrete analogue of inequality (3.15)

$$
\sum_{n=1}^{\infty} n^{\alpha-2 p}\left(\sum_{k=1}^{n} a_{k}\right)^{p} \leq C \sum_{n=1}^{\infty} n^{\alpha}\left(\Delta^{(2)} a_{n}\right)^{p}
$$

which will be satisfied if and only if

$$
B_{17}=\sup _{n}\left(\sum_{n=k}^{\infty} n^{\alpha-2 p}\right)^{1 / p}\left(\sum_{k=1}^{n} k^{\alpha+p^{\prime}(1-\alpha)}\right)^{1 / p^{\prime}}<\infty
$$

and

$$
B_{18}=\sup _{n}\left(\sum_{k=1}^{n} k^{\alpha-p}\right)^{1 / p}\left(\sum_{n=k}^{\infty} n^{\alpha\left(1-p^{\prime}\right)}\right)^{1 / p^{\prime}}<\infty
$$

which is essentially new.

Remark 3.7 As a consequence to inequality (3.15), if we take $\mathbb{T}=\mathbb{R}, \omega(x)=v(x)=e^{\alpha x}$ for the case $p=q=2$ with boundary condition $u(0)=u^{\Delta}(0)=0$ and for $\alpha<0$, we will obtain 
the the following continuous weighted inequality:

$$
\int_{0}^{\infty}|u(x)|^{2} e^{\alpha x} d x \leq C \int_{0}^{\infty}\left|u^{\prime \prime}(x)\right|^{2} e^{\alpha x} d x
$$

which will be satisfied if and only if

$$
B_{20}=\sup _{x \in[0, \infty)}\left(\int_{x}^{\infty} e^{\alpha t} d t\right)^{1 / 2}\left(\int_{0}^{x} e^{\alpha t\left(1-p^{\prime}\right)} d t\right)^{1 / 2}<\infty .
$$

This result is due to Kufner [20] (see also Opic and Kufner [28]).

\section{Acknowledgements}

Not applicable.

\section{Funding}

This research received no external funding.

Availability of data and materials

Not applicable.

\section{Competing interests}

The authors declare that they have no competing interests.

\section{Authors' contributions}

All authors contributed equally to the writing of this paper. All authors have read and agreed to the published version of the manuscript.

\section{Author details}

${ }^{1}$ Department of Mathematics, Faculty of Sciences, Galala University, Galala New City, Egypt. ${ }^{2}$ Department of Mathematics, Faculty of Science, Mansoura University, Mansoura, Egypt. ${ }^{3}$ University of Technology and Applied Sciences-ALRustaq, 329- Rustaq, Sultanate of Oman. ${ }^{4}$ Department of Mathematics, Faculty of Science, Fayoum University, Fayoum, Egypt.

\section{Publisher's Note}

Springer Nature remains neutral with regard to jurisdictional claims in published maps and institutional affiliations.

Received: 23 February 2021 Accepted: 21 May 2021 Published online: 03 June 2021

\section{References}

1. Agarwal, R.P., Bohner, M., Saker, S.H.: Dynamic Littlewood-type inequalities. Proc. Am. Math. Soc. 143(2), 667-677 (2015)

2. Andersen, K.F., Heinig, H.P.: Weighted norm inequalities for certain integral operators. SIAM J. Math. Anal. 14, 834-844 (1983)

3. Anderson, K., Muckenhoupt, B.: Weighted weak type Hardy inequalities with applications to Hilbert transforms and maximal functions. Stud. Math. 72, 9-26 (1982)

4. Beesack, P.R.: Hardy's inequalities and its extensions. Pac. J. Math. 11(1), 31-61 (1961)

5. Bennett, G.: Some elementary inequalities. Q. J. Math. Oxf. Ser. (2) 38(2), 401-425 (1987)

6. Bibi, R., Bohner, M., Pecaric, J., Varosanec, S.: Minkowski and Beckenbach-Dresher inequalities and functionals on time scales. J. Math. Inequal. 7(3), 299-312 (2013)

7. Bohner, M., Peterson, A.: Dynamic Equations on Time Scales: An Introduction with Applications. Birkhäuser, Boston (2001)

8. Bohner, M., Peterson, A.: Advances in Dynamic Equations on Time Scales. Birkhäuser, Boston (2003)

9. Bradley, J.S.: Hardy inequality with mixed norms. Can. Math. Bull. 21(4), 405-408 (1978)

10. Copson, E.T.: Note on series of positive terms. J. Lond. Math. Soc. 3, 49-51 (1928)

11. Gurka, P.: Generalized Hardy's inequality. Čas. Pěst. Mat. 109(2), 194-203 (1984)

12. Hall, R.R.: A Wirtinger type inequality and the spacing of the zeros of the Riemann zeta-function. J. Number Theory 93, 235-245 (2002)

13. Hall, R.R.: Generalized Wirtinger inequalities, random matrix theory, and the zeros of the Riemann zeta-function. J. Number Theory 97, 397-409 (2002)

14. Hall, R.R.: Large spaces between the zeros of the Riemann zeta-function and random matrix theory. J. Number Theory $109,240-256(2004)$

15. Hardy, G.H.: Notes on some points in the integral calculus (LX). An inequality between integrals. Messenger Math. 54, $150-156(1925)$

16. Hardy, G.H., Littlewood, J.E., Polya, G.: Inequalities, 2nd edn. Cambridge University Press, Cambridge (1952)

17. Heinig, H.P.: Weighted norm inequalities for certain integral operators II. Proc. Am. Math. Soc. 95, 387-395 (1985) 
18. Hilscher, R.: A time scales version of a Wirtinger-type inequality and applications. J. Comput. Appl. Math. 141, 219-226 (2002)

19. Hinton, D.B., Lewis, R.T.: Discrete spectra criteria for singular differential operators with middle terms. Math. Proc Camb. Philos. Soc. 77, 337-347 (1975)

20. Kufner, A.: Some remarks concerning the Hardy inequality, function spaces, differential operators and nonlinear analysis. Teubner-Texte Math. 133, 290-294 (1993)

21. Kufner, A.: Higher order Hardy inequalities. Collect. Math. 44, 147-154 (1993)

22. Kufner, A.: A remark on kth order Hardy inequalities. Tr. Mat. Inst. Steklova 248, 144-152 (2005)

23. Kufner, A., Heinig, H.P.: Hardy's inequality for higher order derivatives. Tr. Mat. Inst. Steklova 192, 105-113 (1990)

24. Kufner, A., Maligranda, L., Persson, L.E.: The Hardy Inequalities: About Its History and Some Related Results. Vydavatelski Servis Publishing House, Pilsen (2007)

25. Kufner, A., Persson, L.E.: Weighted Inequalities of Hardy Type. World Scientific, Singapore (2003)

26. Muckenhoupt, B.: Hardy's inequality with weights. Stud. Math. 44(1), 31-38 (1972)

27. Oguntuase, J.A., Persson, L.E.: Time scales Hardy-type inequalities via superquadracity. Ann. Funct. Anal. 5(2), 61-73 (2014)

28. Opic, B., Kufner, A.: Hardy-Type Inequalities. Pitman Research Notes in Mathematics Series. Longman, Harlow (1990)

29. Peňa, S.: Discrete spectra criteria for singular difference operators. Math. Bohem. 124(1), 35-44 (1999)

30. Řehăk, P.: Hardy inequality on time scales and its application to half-linear dynamic equations. J. Inequal. Appl. 5, 495-507 (2005)

31. Saker, S.H.: Hardy-Leindler type inequalities on time scales. Appl. Math. Inf. Sci. 8(6), 2975-2981 (2014)

32. Saker, S.H., Graef, J.: A new class of dynamic inequalities of Hardy's type on time scales. Dyn. Syst. Appl. 23, 83-93 (2014)

33. Saker, S.H., Mahmoud, R.R., Peterson, A.: Weighted Hardy-type inequalities on time scales with applications. Mediterr. J. Math. 13(2), 585-606 (2014)

34. Saker, S.H., O'Regan, D.: Extensions of dynamic inequalities of Hardy's type on time scales. Math. Slovaca 65(5), 993-1012 (2015)

35. Saker, S.H., O'Regan, D.: Hardy and Littlewood inequalities on time scales. Bull. Malays. Math. Sci. Soc. 39(2), 527-543 (2016)

36. Saker, S.H., O'Regan, D., Agarwal, R.P.: Some dynamic inequalities of Hardy's type on time scales. Math. Inequal. Appl. 17, 1183-1199 (2014)

37. Saker, S.H., O'Regan, D., Agarwal, R.P.: Generalized Hardy, Copson, Leindler and Bennett inequalities on time scales, Math. Nachr. 287(5-6), 686-698 (2014)

38. Saker, S.H., O'Regan, D., Agarwal, R.P.: Dynamic inequalities of Hardy and Copson types on time scales. Analysis 34 391-402 (2014)

39. Saker, S.H., O'Regan, D., Agarwal, R.P.: Littlewood and Bennett inequalities on time scales. Mediterr. J. Math. 12, 605-619 (2015)

40. Saker, S.H., Rezk, H.M., Krnić, M.: More accurate dynamic Hardy-type inequalities obtained via superquadraticity. Rev. R. Acad. Cienc. Exactas Fís. Nat., Ser. A Mat. 113(3), 2691-2713 (2019)

41. Saker, S.H., Saied, A.I., Krnić, M.: Some new weighted dynamic inequalities for monotone functions involving kernels. Mediterr. J. Math. 17(2), 1-18 (2020)

42. Saker, S.H., Saied, A.I., Krnić, M.: Some new dynamic Hardy-type inequalities with kernels involving monotone functions. Rev. R. Acad. Cienc. Exactas Fís. Nat., Ser. A Mat. 114, 1-16 (2020)

43. Sinnamon, G.: Kufner's conjecture for higher order Hardy inequalities. Real Anal. Exch. 21(2), 590-603 (1995/96)

44. Stepanov, V.D.: Two-weighted estimates of Riemann-Liouville integrals. Izv. Akad. Nauk SSSR, Ser. Mat. 54(3), 645-656 (1990)

\section{Submit your manuscript to a SpringerOpen ${ }^{\circ}$ journal and benefit from:}

- Convenient online submission

- Rigorous peer review

- Open access: articles freely available online

- High visibility within the field

Retaining the copyright to your article

Submit your next manuscript at $\gg$ springeropen.com 